\title{
ATMOSPHERIC DIFFUSION AND AIR POLLUTION
}

A $\mathrm{N}$ international symposium on "Atmospheric Diffusion and Air Pollution", arranged jointly by the International Union of Theoretical and Applied Mechanics and the International Union of Geodesy and Geophysics, was held in Oxford during August 24-29. The symposium brought together from various countries nearly one hundred scientists, mainly meteorologists and fluid dynamicists, with active interests in one aspect or another of the physics of turbulent diffusion. The scientific programme was planned by a committee under the chairmanship of Sir Geoffrey Taylor and the joint secretaryship of Dr. F. N. Frenkiel (Johns Hopkins University) and Prof. P. A. Sheppard (Imperial College of Science and Technology, London), and was expressly designed to examine outstanding basic problems in the study of atmospheric diffusion and its application to air pollution studies. Accordingly, after an opening session which included broad surveys of the distribution and effects of the airborne products of combustion, conventional industrial processes and nuclear processes, the proceedings were organized into sessions dealing with specific aspects of the problem of diffusion. These included the structure of atmospheric turbulence, the theoretical treatment of the diffusion of particles in a turbulent atmosphere, the processes of vertical transfer on all scales in the atmosphere and the effect of thermal stratification thereon, and the distribution of airborne material from artificial sources.

As nearly forty papers were presented, this article would be little more than a catalogue if all were to be mentioned individually. For such detail the reader will shortly be able to turn elsewhere, for the entire proceedings of the symposium, both papers and discussions, are to be published in book form by Academic Press, Ltd., and it is hoped that they will appear before the end of the year. The aim of this article is rather to comment on some of the major issues brought out in the proceedings

The scientific treatment of atmospheric diffusion was effectively started by Sir Geoffrey Taylor in 1915 in his classical work on the vertical transfer of heat and momentum by eddies in the atmosphere. This work was the beginning of one of the two main approaches to the subject, the approach which is concerned with the 'bulk' properties of the fluid, especially the gradients of the mean properties undergoing diffusion, as distinct from the other main approach involving preoccupation with the internal structure of the fluid. The real beginning of this latter approach was also provided by Taylor, in 1921, in a paper in which diffusion was related to the correlation between the eddy velocities of a given element of fluid at specified times. Then in 1926 L. F. Richardson put forward a novel theory of atmospheric diffusion which implicitly recognized the existence and importance of a whole spectrum of eddy sizes in turbulent motion. More explicit use of the idea of a spectrum came later, at first in a purely formal sense, in the general theory of the statistical mechanics of isotropic turbulence, initiated by Taylor in 1935 and followed up more recently by G. K. Batchelor and others. From a physical point of view, an important step in the development of the concept came with A. N. Kolmogoroff's set of simplifying hypotheses concerning the transfer of turbulent energy from large eddies to small eddies. Although these ideas were put forward in 1941, owing to the Second World War they did not begin to be widely known until nearly ten years later. The fundamental ideas involved in these earlier advances still dominate the background against which the Oxford discussions were conducted.

The contributions on the structure of atmospheric turbulence all indicated the tendency, which has grown rapidly, especially over the past five years or so, to represent this turbulence on the statistical basis which has been so highly developed in the study of wind-tunnel turbulence. Owing to the large range of eddy sizes normally present in the atmosphere, the emphasis has naturally been more on the spectrum of eddy energy than on the timeor space-correlations of the velocity fluctuations, though in practice the time-correlations (from velocity observations at a fixed point) are obtained as an essential intermediate step. The 'power-spectra' derived from them by Fourier transform techniques give the intensities of the contributions, to the total variance of the velocity component, from oscillations of particular frequencies which are contained in the time-variation of the component. These 'timespectra' are strictly to be distinguished from the so-called Eulerian 'space-spectra', which are correspondingly derived from simultaneous velocity measurements at many fixed points, and which contain a more satisfactory physical representation of the eddy 'size'. However, it has been customary for some time to assume equivalence of the more easily obtained 'time-spectra' with space-spectra in the direction of the mean motion, and evidence on this continues to accumulate.

Two features which were brought out concerning the shape of the spectrum are especially worthy of mention here. If the spectra for the vertical component at heights varying from one to a few hundred metres are 'normalized', by plotting the product of the equivalent wave-number (the reciprocal of the wave-length) and the spectral intensity against the product of the wave-number and the height, there appears to be a general similarity in shape, with a maximum intensity at a wave-length of the order of three times the height above ground. The other features concern behaviour at higher frequencies, where, according to the ideas put forward by Kolmogoroff, all forms of turbulence should exhibit isotropic properties, that is to say, properties which are unaffected by rotation of the axes of reference. The data now accumulating leave no doubt that the limiting eddy size, below which the isotropic structure exists, increases with increasing height above ground, and recent measurements confirm an earlier suggestion that the isotropic limit occurs at an equivalent wave-length approximately equal to the height. Both of these generalizations may well require some qualification when appreciable thermal stratification of the atmosphere is involved, but they represent useful 


\section{№. 4643 October $25,1958 \quad$ N A T U R E}

first steps in the provision of an integrated description of atmospheric turbulence.

In the papers relevant to the vertical transfer of momentum and heat in the lower atmosphere, it was encouraging to see the Navier-Stokes equations of fluid motion being used as a starting point for understanding some of the stages in the complex cycle of energy exchange in stabilized and unstable flow. On the other hand, it was evident that some confusion still exists in the empirical description of the effects of thermal stratification. All seems reasonably straightforward so long as the atmosphere is in neutral or near-neutral equilibrium, when the adoption of the familiar universal wind profile (wind velocity varying with the logarithm of the height) enables the flux of momentum (and of other properties) to be usefully estimated merely from measurements of the vertical gradients of wind velocity and of the prop. erties concerned. The long-required extension of this sort of treatment to the cases when the atmosphere is heated or cooled from below has recently seemed to be fortheoming from dimensional analysis. These treatments certainly lead to wind profiles of the required shape in stable and unstable conditions, and also to the correct qualitative variation of vertical flux with degree of stability, but at this point the consistency appears to end. In the first place, there are important differences, which have yet to be explained, in independent measurements of the vertical transfer of momentum near the ground. Furthermore, it seems that the magnitudes of the dimensionless parameters required by the form of the wind profile are of a different order from those required to explain the absolute magnitude of the vertical flux. Since one of the practical interests lies in being able to estimate flux quantities from profile measurements, these discrepancies are frustrating as well as puzzling, and their resolution would represent an advance of some practical importance.

It may be that the resolution of these difficulties will only come as a result of closer attention to the structural properties, as in one of the contributions, where for two specific cases with unstable conditions it was demonstrated that the vertical motions responsible for heat transfer were of larger scale than those responsible for momentum transfer. Moreover, from a number of points there are reminders that with increasing instability and with increasing height above the surface boundary the dominant character of the vertical motion changes from that of mechanically generated turbulence to the more organized motions of thermal convection. Some progress has already been achieved in the representation of the heat transfer by these organized motions, but a useful statistical derivation of the transfer of momentum and other properties has evidently yet to emerge.

In the theoretical treatment of the distribution of particles released in a turbulent fluid increasing use is now being made of the mathematical and statistical techniques which were evolved largely in relation to aerodynamic problems. Many of the developments are still in a highly formalized stage, and the progress made towards rigorous solutions which can be expressed in terms of measurable properties of the turbulence is not immediately obvious. However, one real improvement is to be noted in the more detailed attention which is being paid to the significance of the finite time involved in observing diffusion phenomena. The need for this attention arises directly from the 'width' of the turbulence spectrum, and becomes the more pressing in the case of atmospheric flow, where for many problems the spectrum extends effectively indefinitely into the region of long wave-lengths. Another welcome sign is the increasing recognition of the distinction between the distribution of particles relative to fixed axes after continuous release from a fixed point, and the relative spread of particles released instantaneously in a cluster. These ideas are being adopted to an encouraging degree in a 'model' treatment of the statistical properties of the continuous plume from a fixed source of particles. The accumulation of satisfactory observations on the relative spread of particles is now an outstanding requirement.

Despite these more advanced exploitations of the statistical theories of turbulence, a central problem still remains in the interpretation of the Lagrangian variations of the motion, that is to say, the variations in the velocity of a particle in contrast to the variations which may easily be observed at fixed points in the fluid. This problem was presented from the beginning in Taylor's 1921 analysis, by which the diffusion from a serial release of particles is specified in terms of the Lagrangian correlation coefficient, namely, that between the velocities of a particle at successive instants of time. The gap between this virtually unmeasurable property and the measurable time or space correlations at fixed points has yet to be bridged, and the fluid dynamicists are not yet able to offer any hope of this being achieved soon in the circumstances of concern to the meteoro. logist.

In the absence of a tractable analytical relation between these Lagrangian and Eulerian properties the realization of the practical end-point in the present subject, that is, the formation of realistic estimates of the distribution of airborne material released in a specified fashion, continues to lean heavily on empirical devices or careful limitation of the physical conditions involved. The latter recourse seems to be used with promising effect in contributions to the diffusion of falling particles with terminal velocities of a limited range, large enough to ensure that Eulerian variations are dominant over Lagran. gian variations, yet small enough to exclude serious inertial effects on the response of the particles to the turbulent fluctuations. For floating particles some immediate progress is provided by the empirical step of assuming a simple relation between the Lagrangian correlation function and the auto-correlation function measurable at a fixed point. This has led to a method of obtaining the spread from a continuous point source directly and simply from measurements of velocity fluctuations.

The attainment of rational, albeit empirical, formulæ and methods for predicting diffusion is still mainly confined to the small-scale processes involved in diffusion over a few hundred metres. On the largest scale, involving exchange within and between the troposphere and stratosphere, the position is still one of gaining experience from the behaviour of natural and man-made tracer elements. Much interesting information is accumulating from measurements of fall-out from nuclear tests, and some of this is thought to fit in with current ideas of stratospheric motion based on studies of water vapour and ozone. Another promising radioactive tracer has been recognized in the isotopes produced by cosmic radiation. These have the advantage of being produced practically independently of time and without seasonal or diurnal changes. For the inter- 
mediate scale of diffusion, the sort involved in the travel of radioactive material from the Windscale reactor accident in October 1957, little progress has been reported. On this scale, involving heights of several thousands of feet and distances of tens of miles, the processes of dispersal are further complicated on one hand by the variation of the mean wind field with height, and on the other hand by the large-scale effects of thermal stratification, leading alternatively to the virtually complete suppression of vertical spread or to rapid ascent in organized convective motion. From a practical point of view, it is to this scale of processes that attention is most urgently required.
F. Pasquill

\section{FUTURE OF MINERAL EXPLORATION IN GREAT BRITAIN AND IRELAND}

$\mathrm{T}$ HE fourth Sir Julius Wernher Memorial Lecture of the Institution of Mining and Metallurgy was given by Mr. Anton Gray, mining adviser to the U.K. Atomic Energy Authority, at the Royal Institution on September 22, his subject being "The Future of Mineral Exploration". In a survey of the world situation, Mr. Gray maintained that whereas it is unlikely that the present rapid rate of increase of consumption of metals can be maintained, it would not be surprising if the demand for the major metals doubles before the end of the present century. About the beginning of this century the geologist began to take over the work of the prospector, for by then most of the mineral deposits not concealed by younger formations had been found. But he has not had the same success in the search for a method of predicting the occurrence of ore-bodies as his colleague in the petroleum industry has had in finding con. cealed oil-pools.

This results from his inadequate knowledge of the precise mode of origin of mineral concentrations ; for while it is generally agreed that most important ore-bodies were introduced into openings in preexisting rocks by warm waters, there is no agreement as to the source of the water or of the contained metals. The dominant hypothesis, that of hydrothermal solutions related to granite, had been shown, in Mr. Gray's view, to be adequate only for a limited group of metals, including tin, tungsten, beryllium and some gold concentrations. He suggested an unorthodox but not heretical alternative for the other metals : that the waters are generated during metamorphism associated with mountain-building, and he based this suggestion on two of the great ore deposits of the world, the central African copper belt and the Witwatersrand goldfield. The great part which Mr. Gray has played in mineral exploration in central Africa lends authority to his advocacy of this hypothesis of what might be called metamorphic lateral secretion.

This lecture formed a prelude to a symposium on "The Future of Non-Ferrous Mining in Great Britain and Ireland", held at the Federation of British Industries on September 23-24. The symposium consisted of twenty-four papers, in three broad groups : a series of seventeen devoted to descriptions of the geology, mining history and potentialities of the mineralized districts of Ireland, Scotland, England and Wales; a group of short contributions on technical aids to mineral exploration, such as the various geophysical methods, the use of geochemical prospecting techniques and of air photography ; and a series on economics and finance.
The foundation for an approach to the geological problems of the mineral fields was laid during the past century by the official primary 6-in. Geological Survey of Great Britain and Ireland, but with the decline of the tin, copper and lead mines, there was little further interest in the subject until recently. The Special Reports on Mineral Resources of the Geological Survey, chiefly based on work done during the First World War, brought together much valuable data, but it is probably fair to say that geological investigation by modern methods has been confined to the past two decades. The papers in the first group gave a fair conspectus of the conclusions reached. As is well known, the mineral fields in the British Isles occur in granite and adjacent DevonianCarboniferous slate in the south-western counties of England; and elsewhere chiefly in two main environments, the Lower Palæozoic slates and very low-grade metamorphics; and the unmetamorphosed Lower Carboniferous rocks, chiefly of limestone or 'Yoredale' facies. A stratigraphical control--that is to say, a strict relationship between particular beds or lithologies and introduced mineral bodies-is postulated by W. J. Hughes in central Wales, by A. A. Archer in north Wales, and by $H$. G. Dines in west Shropshire, in all these cases in Lower Palæozoic strata; but in other districts where the rocks are of similar age, such as the Lake District (T. Eastwood) or Leadhills-Wanlockhead (R. A. Mackay), no such control has been found. Thus, while the ore-shoots, though steeply inclined, tend to be elongated ribbons where they are related to the stratigraphy, where they are not-as, for example, in the Greenside lead or Coniston copper mines-the two major dimensions tend to be roughly equal. In the Carboniferous fields of the Pennines and Flint-Denbigh there is a very well-marked relation to brittle beds such as sand. stone and limestone, as well as to chemically reactive wallrocks such as limestone and dolerite; and the influence of impermeable or semi-permeable shales or rotted lavas overlying favourable strata is important in localizing ore-bodies. It follows that in these districts, as well as in many Lower Palæozoie fields, ore-bodies occur which fail to outcrop. Some of these have been found by underground operations, but nearly all districts contain areas suitable for prospecting for concealed mineral deposits, some of them of substantial promise. This is the ehief basis for the view that British metalliferous deposits are far from exhausted.

A second line of geological approach is the study of the spatial distribution of mineral species in individual districts. The zonal pattern in Cornwall 